\title{
Some methodological considerations in thermal biofeedback training
}

\author{
EDWARD TAUB and PAUL J. SCHOOL \\ Institute for Behavioral Research, 2429 Linden Lane, Silver Spring, Maryland 20910
}

\begin{abstract}
This article attempts to provide guidelines for acceptable practice in thermal biofeedback training. Criteria are set forth in three major areas: the nature of the interaction between the experimenter/therapist and subject/patient, training procedures, and the physical characteristics of the temperature sensing and feedback system.
\end{abstract}

In thermal biofeedback training, as in almost all other types of biofeedback training, it is of the greatest importance that three major factors be given explicit attention: (1) the nature of the interaction between the subject/patient and experimenter/therapist, (2) adequate methodological procedures to insure both that the training process is as easy as possible and that a true training effect and not artifact is being recorded, and (3) the physical characteristics of the training environment and of the sensing, feedback, and recording system. It is beyond the purview of this article to deal with each of these subjects exhaustively; however, we will attempt to specify those aspects of thermal biofeedback procedures and techniques that are the most important for avoiding artifact and achieving a good result in training.

\section{THE "PERSON FACTOR" IN THERMAL BIOFEEDBACK TRAINING}

Perhaps the most powerful factor influencing whether or not thermal biofeedback learning will occur is the quality of the interaction between the experimenter/ therapist and the subject/patient, that is, the "person factor." The first experimenter carrying out thermal biofeedback research in our laboratory (May 1970) showed an impersonal attitude toward the experimental subjects and was able to train only 2 of 22 individuals to control skin temperature. Another experimenter, using exactly the same technique, was more informal and friendly, and trained 19 of 21 subjects. We assumed that the person factor was responsible for this apparent reversal of results.

This was our first observation along these lines, and it

This research was supported by NIH Grant HL 21323 and by ARPA of DOD under ONR Contract N00014-70-C-0350 to the San Diego State College Foundation. We thank Dr. Joseph Rothberg for the design and construction of the feedback system and Maurice Swinnen for the design and construction of the thermistor probes and thermistor bridges of the first analog temperature training device employed in this laboratory. Alfred Jaknuinas designed and built our current digital temperature system. We are grateful to our collaborators who helped in the conduct of different stages of the research, in particular, Paul Slattery, Cleeve S. Emurian, Priscilla Howell, and Susan N. Rice. was admittedly unsystematic. A formal experiment was carried out to investigate this phenomenon. The independent variable was the experimenter's behavior toward two groups of subjects. With one group the experimenter adopted an impersonal attitude (i.e., using last names, discouraging extraneous conversation, avoiding eye contact, etc.). With another group the experimenter adopted a friendly attitude (i.e., using first names, encouraging development of a friendly relationship, frequent eye contact, etc.). Both groups showed significant learning. However, the impersonally treated group altered hand temperature by a mean of only $1.3^{\circ} \mathrm{F}$ on the last 3 days of a 10-day training series; the group treated in a friendly manner achieved a mean change of $4.2^{\circ} \mathrm{F}$ on the same days. This striking difference is by far the largest experimental effect we have obtained by the manipulation of any single variable in our entire sequence of experiments.

It is almost impossible to overemphasize the importance of the experimenter-attitude variable for the success of thermal biofeedback training. It seems highly probable that the person factor is equally critical for the success of other types of biofeedback training.

\section{ADEQUATE METHODOLOGICAL PROCEDURES}

\section{Presession Stabilization Procedures}

It is extremely important to have an initial stabilization period prior to the beginning of a feedback period. It is well known that skin temperature sensitively reflects the emotional state of the individual (e.g., Mittelmann \& Wolff, 1939; Neuman, Lhamon, \& Cohn, 1944). When a subject is involved in a negative affective state, his hands tend to be cold; as he relaxes, his hands tend to increase in temperature. At the beginning of a session, skin temperature can change enormously; when the change is large, it is usually in an upward direction. The large increases are apparently an indicator that the subject is relaxing after being attached (wired up) to the equipment and left alone. Laboratory situations are tense for most people, so subjects often do not relax until the experimenter leaves the room. It is important not to confuse the effects of general relaxation with the 
effects of specific training. The training task should not be introduced until the relaxation process has been completed. We have been shown results by investigators new to the field that purport to indicate major temperature self-regulation effects on a few individual training days but not on others. These results often arouse suspicion since, once a person has learned the task well, he is able to repeat it on almost all occasions. Often novice investigators do not establish initial stabilization periods. Almost certainly, the result is the type of habituation process described above.

It is preferable to use a stabilization procedure based on performance rather than the simple passage of time. In this laboratory the initial stability criterion established before feedback training requires that the temperature variation be no greater than $.25^{\circ} \mathrm{F}$ during 4 consecutive min. The first $6 \mathrm{~min}$ are not considered part of the period used to determine the initial stability point; that is, training cannot begin until at least $10 \mathrm{~min}$ have elapsed. If the stability criterion is not reached in $30 \mathrm{~min}$, self-regulation is initiated. However, this occurs infrequently. The mean time required for the initial stability point to be reached differs with the season of the year, but is usually about $15 \mathrm{~min}$. Examination of session records in this laboratory indicates that, had a standard temporal interval (such as $15 \mathrm{~min}$ ) been employed, many subjects would already have stabilized. However, some subjects would not have stabilized, thus contaminating the data for the training period.

\section{Pretraining Baseline Sessions}

Some individuals have a marked tendency to exhibit temperature change in a given direction while simply sitting quietly in an experimental chamber for a period of time equivalent to the full biofeedback session. This does not occur in every individual. The temperature of some individuals' hands increases during some baseline sessions and decreases during others. However, when there is a tendency to change in a single direction, it is important that this be known, since it can make a major alteration in the interpretation of the amount of learning that has taken place. The manner in which this problem has been handled quantitatively in this laboratory is described elsewhere (Taub, 1977; Taub \& Emurian, 1976).

The tendency for temperature change in a given direction is most marked during the summer. People tend to come into the experiment from the street with very high hand temperatures, and these frequently do not decrease even after sitting in an air-conditioned laboratory for $1 \mathrm{~h}$ before the beginning of a session. During the stabilization period, there is an almost invariable tendency for the hand temperature to decrease slightly, though it often remains above $94^{\circ} \mathrm{F}$. This is a difficult problem to overcome. We had subjects wash their hands in cool water, but this often produced an instability in hand temperature over a long period of time. Moreover, hand temperature often returned to the original high level within $1 / 2 \mathrm{~h}$ or so. In these cases, we simply accept the initial high hand temperature and proceed with the training from that point. Because outside temperature can be such an important factor in influencing subjects' starting temperatures and the results obtained in the biofeedback situation, we routinely record the outside temperature prior to each session.

At present, we carry out 5 days of baseline testing. Data for the first day are discarded since the initial period of habituation to the laboratory frequently gives an atypical record. This procedure is appropriate because most of our work is experimental. For clinical purposes, baseline sessions are probably not absolutely necessary. However, it is extremely important that there be an initial stabilization period, regardless of setting.

\section{Methods for Determining Whether the Result is Effect or Artifact}

One of the best methods for determining that true learning has taken place is to train a subject to alter his temperature in both an upward and downward direction. This can be done in serial periods during the same session from the beginning of training or it can be done sequentially, that is, the subject learns to control temperature in one direction and then in the other. If the subject uses a "trick" to accomplish the task, he probably cannot use the same trick to change temperature in the opposite direction. It is possible, of course, but it is highly improbable that the subject can use two tricks with equal effectiveness. If a bidirectional procedure is employed, considerations of stabilization periods and baseline sessions become less important than if the subject is trained in only one direction. Most clinicians, of course, teach patients to warm their hands, a unidirectional procedure. Thus, bidirectional training is inappropriate for clinical purposes. However, steps can be taken to reduce the chances of artifacts influencing the procedure. One method is to give the subject appropriate instructions prior to the beginning of training. $\mathrm{He} / \mathrm{she}$ should be told to move as little as possible, compatible with comfort; this is especially true for moving the hands. The subject should also be told to avoid changing breathing from normal resting patterns. During the session, it is important to have subjects under visual observation by the experimenter. If subjects deviate from instructions, they should be requested to stop the undesirable maneuvers, by intercom if subject and experimenter are in separate rooms.

The temperature recorded by thermistors can be altered by blowing on the devices. Some subjects direct expired breath in the direction of the self-regulating hand without being aware of it. When the phenomenon of feedback-aided self-regulation of hand temperature was first demonstrated in this laboratory, we con- 
structed a transparent Lucite box, open in the direction facing away from the subject to permit circulation of air. On the side nearest the subject, there was an opening into which the hands fit; a drop cloth covered the opening. This device permitted us to determine that the passage of expired air over thermistors was not a factor in the control we were observing in our subjects at the time. Clinicians should be alert to this possibility and, when appropriate, instruct subjects to refrain from such activities. In order to prevent heat from being trapped around the thermistors, thus generating unduly high readings, a framed screen (Chucker, Fowler, Motomiya, Singh, \& Hurley, 1971) rather than a solid wooden board can be used as a lap board upon which the hands rest.

The training period in this laboratory is typically $15 \mathrm{~min}$, although some subjects given feedback for 15 consecutive $\mathrm{min}$ complain of fatigue. Maintaining focused attention for that long can be aversive for some people. Consequently, we have introduced a $10-\mathrm{sec}$ timeout period between successive $50-\mathrm{sec}$ feedback periods. We have no systematic data on this issue, but when we introduced the 10-sec between-trial interval, the subjects uniformly reported a preference for that procedure rather than for continuous feedback.

\section{Posttraining Rest Period}

It is useful to have a posttraining rest period. This procedure serves at least two purposes. First, if the subject is achieving self-regulatory control by employing "passive volition" (Green, Green, \& Walters, 1970), it is valuable to give the subject an opportunity to emerge slowly from that condition. If subjects begin to engage in normal activity too soon, they sometimes report discomfort and mild headache. Second, if a subject is being taught to alter temperature in one direction only, temperature variation during a posttraining rest period provides a useful crude index of how well the subject has learned the task. In the early stages of learning, temperature does not change rapidly from the level attained at the end of the feedback period. When learning is well established, however, there is a marked tendency for the temperature to return to baseline fairly rapidly.

\section{The Information Display}

Two types of visual feedback displays have been employed in this laboratory. The first was an analog device that controlled the brightness of a lamp so that it was proportional to the temperature at a feedback locus on the dominant hand (Taub \& Emurian, 1976). A priori considerations and comments from subjects indicated that this feedback system had a number of drawbacks; consequently, a more sophisticated digital display was constructed (Taub, 1977). Surprisingly, the presumably superior feedback system did not improve the ability to self-regulate hand temperature (for a fuller discussion, see Taub, 1977). Roberts and co-workers (Roberts, Kewman, \& Macdonald, 1973; Roberts, Schuler, Bacon, \& Zimmerman, 1975) have employed auditory feedback and have obtained results similar to those of this laboratory.

\section{Use of Other than Augmented Feedback}

A variety of feedback techniques other than that obtained from temperature sensors, which is processed electronically and then displayed, can greatly improve a subject's performance. Verbal reward for a subject who does well is extremely important. When the subject is not doing well, encouragement and an implicit attitude that the experimenter confidently believes that he will be able to learn the task are even more important. For the subject who is doing poorly, even small movements in the correct direction should be praised.

When training college students, we routinely employ monetary reward at the rate of 25 cents for each $.25^{\circ} \mathrm{F}$ in the correct direction. In one study this procedure improved performance by $.5^{\circ} \mathrm{F}$, but the difference between subjects given money reinforcement and those without it was not statistically significant. We have continued the money reinforcement procedure because it seems to focus a subject's attention on the task.

At the end of each session, the subject is given a graph showing his performance on that day. This process can be an important ancillary device for maintaining subject interest and motivation.

Finally, at the beginning of training we do not permit a subject to receive too much feedback on changes in the wrong direction; $.2^{\circ} \mathrm{F}$ is the maximum allowed. When subjects observe large changes in the wrong direction, many become discouraged and stop trying or become anxious and try too hard. Both reactions are counterproductive and usually result in an inability to perform. The negative results are sometimes not confined to that day alone, but generalize and lead to a complete failure of training.

\section{Units of Feedback and Sampling Rate}

We do not have systematic data on units of feedback and sampling rate. However, at the beginning of our work on this project, feedback was given in units of $.1^{\circ} \mathrm{F}$, and learning was poor. The system was redesigned so that the feedback unit was $.02^{\circ} \mathrm{F}$, and the subsequent results were excellent (Taub \& Emurian, 1976). Evidently, if the feedback unit is greater than $.5^{\circ} \mathrm{F}$, learning is not as great as it could be with many subjects.

Our current digital feedback system has the capability of a broad range of sampling rates. We began with a sampling-display change rate of $4 / \mathrm{sec}$, that is, the feedback display changed four times every second. Subjects found this unpleasant and confusing. We then reduced the sampling-display change rate to $2 / \mathrm{sec}$, and this was greatly preferred. There is obviously a U-shaped function relating sampling rate and thermal biofeedback learning. 
We have only the most general idea of what the parameters are.

\section{Factors Conducive to Subject Relaxation}

A number of simple conditions can generate a relaxing environment. The subject should be seated in a comfortable chair. The light in the room should be dim. It is desirable to have a rug on the floor since this lends a feeling of warmth and ease. The decor should be pleasant. A few plants and prints or paintings in the vicinity seem to help. On the other hand, during the early part of training, stressors tend to abolish the ability to self-regulate hand temperature. After the task has been well learned, even severe stressors, such as immersing the noncontrolling hand in ice water or whole-body cold challenge by water in a "cold suit," do not appreciably affect performance (Taub, 1977).

\section{PHYSICAL CHARACTERISTICS OF ENVIRONMENT AND FEEDBACK SYSTEM}

Several physical and technical factors may influence temperature biofeedback results. Environmental factors include temperature, temperature variability, and air flow patterns in the subject's room, as well as outside temperature. Proper sensor placement is critical. In addition, a number of other technical factors contribute greatly to the validity and reliability of the measurement.

\section{Temperature and Air Flow in the Biofeedback Room}

It is virtually impossible to devise a means of reliably sensing skin temperature that is wholly uninfluenced by air temperature and flow. Air drafts introduce artifactual measurement variation, not only because they are warmer or cooler than the mean ambient temperature but also because they change skin temperature by altering evaporative skin cooling. Strong drafts probably cause so great a spurious moment-to-moment temperature change that acquisition is either considerably slower or prevented entirely. Similar considerations apply to any other factor that produces large or rapid changes in room temperature. Consequently, any temperature variation caused by lack of sufficient thermostatic control or drafts must be corrected.

Typically, home or office room temperatures, which presumably are being maintained at a constant level, vary over a $1.5^{\circ} \mathrm{F}$ to $3.0^{\circ} \mathrm{F}$ range in a given location. The variability is increased still further because most heating/cooling systems produce temperature gradients that vary greatly at different locations in a room.

In our laboratory several steps have been taken to achieve a biofeedback room temperature of $73^{\circ} \mathrm{F} \pm$ $.25^{\circ} \mathrm{F}$. The inside of the room has been insulated with 3-in.-thick fiberglass. The material is left essentially open, being kept in place by decorative burlap stapled to the wall. This arrangement eliminates hot or cold spots on the walls and ceiling. Moreover, the essentially exposed surface of the fiberglass provides excellent acoustical isolation. To facilitate achieving the desired biofeedback room temperature, we maintain the temperature of the large air mass in the entire laboratory at $74^{\circ} \mathrm{F}$, a level slightly above the desired biofeedback room temperature. A portion of this air is drawn into a semiclosed system consisting of a 13,000-Btu window air conditioner that continuously supplies air to the biofeedback room through ducts and registers located in the ceiling. Return air is picked up with a duct at floor level.

The biofeedback room contains a false ceiling constructed of a 1-in.-thick sheet of air-filter material, called Permalast, suspended $20 \mathrm{~cm}$ below the real biofeedback room ceiling. This results in an "air box" with a very porous lower surface. An empirically designed system of baffles within the false ceiling causes inlet air to be fairly uniformly distributed within the space between the false and real ceilings. Although the air flow through the biofeedback room is fairly high, the system of baffles, air filter, and floor-level-returnduct produces a very uniform ceiling-to-floor and wall-to. wall temperature distribution $\left(<.5^{\circ} \mathrm{F}\right)$ with no perceptible air drafts.

A Yellow Springs Instruments Model 73 telethermometer set at $72.75^{\circ} \mathrm{F}$ with a Model 401 probe controls the compressor in the air conditioner. Many probe locations were tried to achieve the least possible temperature variation. Surprisingly, the best system performance was obtained when the probe was placed in a very atypical location-the air conditioner outlet duct. With this arrangement, the compressor comes on for $10.15 \mathrm{sec}$ every $1.2 \mathrm{~min}$. This brief "on time" is effective in smoothing out thermal hysteresis effects.

The level of temperature control in our laboratory is desirable in a thermal biofeedback setting, but it is recognized that this will be difficult, if not impossible, to achieve in a typical clinical setting. However, a number of relatively simple steps can be taken to improve temperature level and stability. First, a check can be made of the temperature in the portion of the room in which the patient will be located. If there are drafts in that area, the location of the patient should be changed. If a location change is not possible, some means of draft deflection, such as room-dividing screens or large leafy plants, should be provided. If the room temperature still varies more than $1.5^{\circ} \mathrm{F}$ during a typical session, qualified service personnel or an air conditioning/heating consultant can recommend changes to reduce temperature variation.

\section{Temperature Sensor Attachment Methods}

Although there are noncontact methods of skin temperature recording such as thermography, most temperature biofeedback is done with sensors attached to the skin surface. Obviously, proper attachment of 
the sensors is required to insure adequate sampling of the physiological variable. To prevent the obstruction of blood flow, our subjects are asked to remove rings and tight wrist bands and not to wear tight sleeves. When sensors are located on the fingers, the tape securing them should not occlude blood flow. This can be avoided by applying the tape with light pressure and covering only a portion of the circumference of the finger. Complete encirclement of the finger should be avoided. Attachment is accomplished with a single layer of "skin tape" (Dermilite). For a technical reason (stem effect, described below), we always tape at least $5 \mathrm{~cm}$ of the sensor lead wire to the subject's skin. The second strip of tape also reduces the chances of a sensor falling off during a session.

\section{Temperature Measurement Sensors and Related Equipment}

Most temperature biofeedback equipment employs thermistor-type sensors. Thermocouple sensors are not as commonly used. Thermistors are semiconductor devices which change electrical resistance when exposed to different temperatures. Thermocouples are made of two unlike metals that produce a voltage when they are connected electrically. When attached to appropriate circuitry, both thermistors and thermocouples provide a voltage analog that is proportional to temperature.

Although thermocouples tend to be inexpensive, fairly linear, and rapid responding devices for skin temperature measurement, most designers of temperature biofeedback equipment have elected to use thermistors because, for the same system cost, the devices provide more sensitive, accurate, and higher resolution readout than that obtainable with thermocouples (The 1977 Omega Engineering Temperature Measurement Handbook, 2nd ed., available from Omega Engineering, Inc., Box 4047, Stamford, Connecticut 06907).

When a commercially available thermilinear device is used to sense temperature, the output voltage is linearly related to temperature. Thermistors do not have a linearizing thermally coupled resistor and are, therefore, not thermilinear devices. Consequently, they may or may not provide enough accuracy, depending upon experimental/clinical requirements. Generally, the small additional expense for thermilinear devices is worth the benefit.

No temperature-measuring device responds to a temperature change instantly. The time a sensor takes to respond is usually described in terms of "time constant." A time constant is defined as the time it takes a sensor to register $63 \%$ of a newly impressed temperature. If a sensor has a time constant of $1 \mathrm{sec}$ and is exposed to a $1^{\circ} \mathrm{F}$ change, the output of the system it drives will indicate $.63^{\circ} \mathrm{F}$ when $1 \mathrm{sec}$ has elapsed, $.86^{\circ} \mathrm{F}$ after $2 \mathrm{sec}$, and $63 \%$ of the remaining difference from $100 \%$ of the full temperature change in each additional second.

Commercially available biofeedback equipment typically incorporates sensors having time constants ranging from approximately $.3 \mathrm{sec}$ to several seconds. At present there are no data indicating that shorter time constants confer an advantage in temperature biofeedback training. Intuition suggests that the shorter time constant is better. However, when one considers that most subjects acquire control of skin temperature fairly rapidly, despite the fact that the time constant of the skin responding to blood-flow changes is on the order of 5 or more seconds, the additional temporal lag produced by typical sensor time constants of .3 to $3 \mathrm{sec}$ may not be important, especially when very high resolution $\left(.02^{\circ} \mathrm{F}\right.$ increments) feedback is provided.

Our laboratory employs thermilinear network sensing devices designed to provide good thermal coupling with the skin, a relatively short time constant $(.9 \mathrm{sec})$, extra electrical insulation to eliminate the possibility of shock hazard, and small-flexible lead wires (Model S015358m, Yellow Springs Instruments).

Accurate skin temperature sensing requires that a sensor be thermally coupled to the skin. To maximize thermal coupling, a sensor should be selected that allows good mechanical contact with the skin. Several temperature measurement specialists have recommended the use of a nonevaporative liquid or viscous substance such as mineral oil, petroleum jelly, or silicone grease to increase thermal coupling. We tested such thermal coupling agents and found they provided no significant advantage. When a perceptible draft was passed over the sensor, sensor wires, and sensed skin area, a drop of mineral oil placed under the sensor eliminated about one-half of the $.5^{\circ} \mathrm{F}-1^{\circ} \mathrm{F}$ change that occurred in the absence of a thermal coupling substance. Since thermal coupling liquids are messy and hard to remove, and since they confer little advantage in a nondrafty room, we have elected not to use them. The need for thermal coupling substances should be evaluated in terms of local needs and situations.

\section{Stem Effect}

Electrical currents produced by sensors must, of course, be carried by wires or leads attached to the sensing element. Since wires are thermally conductive, the temperature of the lead wires influences the temperature of the sensor. This is called "stem effect" and can produce large artifacts in recording skin temperature. When a sensor is attached to the skin and the sensor leads are suspended away from the skin, a $1.5^{\circ} \mathrm{F}$ temperature impressed on the lead wires (not the sensor) produces a $.5^{\circ} \mathrm{F}$ change in readout. Stem effect artifact is reduced to insignificant proportions if at least $5 \mathrm{~cm}$ of the sensor lead wires immediately adjacent to the sensor are thermally coupled to the skin by attachment with skin tape.

\section{Equipment Calibration}

A properly designed temperature biofeedback system should provide an absolute readout accuracy of greater than $\pm .3^{\circ} \mathrm{F}$ in the $68^{\circ} \mathrm{F}$ to $98^{\circ} \mathrm{F}$ range. To assure that 
temperature biofeedback equipment is operating properly, one must perform frequent checks of equipment operation and carry out periodic temperature calibrations. The latter is important because the components and sensors employed in even the best systems change with use and age. In our laboratory, thorough calibrations are carried out every 4 months and each time sensors are changed or equipment is repaired.

Temperatuie calibration requires a means of impressing a stable, accurately determined temperature on the sensor. We utilize a Haake Instruments, Inc., Model FE constant-temperature circulator. If the Haake instrument has been temperature stabilized for $2 \mathrm{~h}$, it will main tain a temperature that is constant within $.05^{\circ} \mathrm{F}$. Readings taken in $2.0^{\circ} \mathrm{F}$ steps are sufficient for an adequate calibration. To insure that a sensor is being exposed appropriately to the bath temperature, it must be immersed $10 \mathrm{~cm}$ into the bath, and it must be located near, but not touching, the analytic grade mercury thermometer used for calibration readout.

We acquired the Haake constant-temperature circulator because our temperature biofeedback equipment is large and elaborate and because our research requires accurate absolute temperature calibration. Clinical temperature biofeedback units are portable; therefore, they may be taken to a commercial calibration laboratory. If the clinician desires, a crude calibration check can be made in his own office through the use of a 1-liter (or larger) beaker filled with water. The water should be gently stirred with a laboratory-type magnetic stirring device. An analytic grade thermometer inserted in the beaker can then be used to determine the accuracy of temperatures recorded by the commercially available biofeedback device.

\section{REFERENCES}

Chucker, F., Fowler, R. C., Motomiya, T., Singh, B., \& HURLEY, W. Induced temperature transients in Raynaud's disease measured by thermography. Angiology, 1971, 22, 580-593.

Green, E. E., Green, A. M., \& Walters, E. D. Voluntary control of internal states: Psychological and physiological. Journal of Transpersonal Psychology, 1970, 2, 1-26.

Mittelmann, B., \& WolfF, H. G. Affective states and skin temperature: Experimental study of subjects with "cold hands" and Raynaud's syndrome. Psychosomatic Medicine, 1939 , 1, 271-292.

Neuman, C., Lhamon, W. T., \& Cohn, A. E. A study of factors (emotional) responsible for changes in the pattern of spontaneous rhythmic fluctuations in the volume of the vascular bed of the fingertip. Journal of Clinical Investigation, 1944, 23, 1-9.

Roberts, A. H., Kewman, D. G., \& Macdonald, H. Voluntary control of skin temperature: Unilateral changes using hypnosis and feedback. Journal of Abnormal Psychology, 1973, 82, 163-168.

Roberts, A. H., Schuler, J., Bacon, J. G., Zimmerman, R. L. \& Patterson, R. Individual differences and autonomic control: Absorption, hypnotic susceptibility, and the unilateral control of skin temperature. Journal of Abnormal Psychology, 1975, 84, 272-279.

TAUB, E. Self-regulation of human tissue temperature. In G. E. Schwartz \& J. Beatty (Eds.), Biofeedback: Theory and research. New York: Academic Press, 1977. Pp. 265-300.

TAUB, E., \& EMURIAN, C. S. Feedback aided self-regulation of skin temperature with a single feedback locus: I. Acquisition and reversal training. Biofeedback and Self-Regulation, 1976, $1,147 \cdot 167$. 